\title{
Prevalence of Dyspepsia in Iran: A Systematic Review and Meta-analysis
}

\author{
Mohammad Karimian, MD¹; Reza Ranjbar, PhD²; Majid Salamati, MD¹; Amir Adibi, MD³; Fatemeh Kazemi, MD; Milad Azami, \\ $\mathrm{MD}^{5^{*}}$ \\ 'Department of General Surgery, Faculty of Medicine, Ilam University of Medical Sciences, Ilam, Iran \\ ${ }^{2}$ Professor of Medical Bacteriology, Molecular Biology Research Center, Systems Biology and Poisonings Institute, Baqiyatallah \\ University of Medical Sciences, Tehran, Iran \\ ${ }^{3}$ Department of Psychiatry, Faculty of Medicine, Ilam University of Medical Sciences, Ilam, Iran \\ ${ }^{4}$ Faculty of Medicine, Qazvin University of Medical Sciences, Ilam, Iran \\ ${ }^{5}$ Faculty of Medicine, Ilam University of Medical Sciences, Ilam, Iran
}

\begin{abstract}
Background: Dyspepsia is a highly prevalent gastrointestinal problem. The present study was carried out to assess the prevalence of dyspepsia in Iran.

Methods: The present study was registered at PROSPERO with the code CRD42019148610. It was carried out based on MOOSE and reporting was performed according to the PRISMA protocol. Systematic search of the literature was performed in July 2019 on international databases of PubMed/Medline, Web of Science (ISI), Cochrane Library, EBSCO, CINAHL, EMBASE, Scopus, Science Direct, and local databases as well as the Google Scholar search engine. Heterogeneity was evaluated using $I^{2}$ and Chi-square tests. All analyses were done using Comprehensive Meta-Analysis software.

Results: Overall, 14 studies with a sample size of 54,118 subjects entered in this meta-analysis. The prevalence of dyspepsia in Iran was $14.6 \%\left(95 \% \mathrm{Cl}\right.$ : 9.6-21.7). Large heterogeneity was detected among studies $\left(I^{2}=99.62 \%, P<0.001\right)$. The prevalence of dysmotility-like, ulcer-like, and unspecified dyspepsia was estimated to be 9.7\% (95\% Cl: 4.9-18.4), 12.1\% (95\% Cl: 5.2-25.7) and $17.0 \%(95 \% \mathrm{Cl}: 7.8-33.4)$, respectively. The prevalence of dyspepsia in Iranian men and women was found at $11.1 \%(95 \%$ Cl: $6.3-18.8)$ and $17.8 \%(95 \% \mathrm{Cl}: 10.0-29.7)$, respectively.

Conclusions: The prevalence of dyspepsia in Iran is relatively high. However, it is lower than global estimates.

Keywords: Dyspepsia, Iran, Meta-analysis, Prevalence

Cite this article as: Karimian M, Ranjbar R, Salamati M, Adibi A, Kazemi F, Azami M. Prevalence of dyspepsia in Iran: a systematic review and meta-analysis. Arch Iran Med. 2021;24(7):568-578. doi: 10.34172/aim.2021.80
\end{abstract}

Received: August 25, 2019, Accepted: January 12, 2021, ePublished: July 1, 2021

\section{Introduction}

Dyspepsia is a very prevalent gastrointestinal disease and its prevalence has been reported to be $40 \%$ in populationbased studies. ${ }^{1}$ The Rome II Criteria for the diagnosis of functional dyspepsia (FD) include: FD for more than twelve weeks, which does not need to be continuous, within the past twelve months with: (a) Constant or repeated symptoms (any kind of discomfort or pain focused in the upper part of the abdomen); (b) No sign of organic illness (on endoscopic examination) that is probable to describe the manifestation; and (c) No sign to demonstrate that FD is just improved by defecation or related to the beginning of a change in form or frequency of stool (i.e., not irritable bowel syndrome [IBS]). FD includes (1) ulcer-like FD: Discomfort or pain concentrated in the epigastric part of the abdomen is the main manifestation (the most annoying); (2) Dysmotility-like FD: A disagreeable or irritating painless sensation concentrated in the epigastric part of the abdomen is the main manifestation; this sensation is perhaps identified by or associated with early satiety, fullness of upper abdominal, nausea or bloating;
(3) Unspecified FD: patients whose manifestation do not meet the prerequisites for dysmotility or ulcer-like FD. ${ }^{2}$ According to the Rome IV criteria, which were recently revised, ${ }^{3} \mathrm{FD}$ is characterized by: (a) Constant or repeated dyspepsia for above three months within the past six months; (b) No sign of a probable organic reason of the endoscopic examination; and (c) No evidence that the dyspepsia is only ameliorated by defecation or any relation with stool abnormalities. This last criterion serves to omit IBS as a possible reason of the manifestation, although about $30 \%$ of patients with FD suffer from IBS.

Although clinical assessment is needed to specify if dyspepsia is FD or structural in a person, endoscopic examination shows that most are FD. FD is a complex issue with probable factors such as visceral hypersensitivity, immune activation, brain-gut axis dysfunction, and delayed gastric emptying and Helicobacter pylori infection. ${ }^{4-8}$

Although FD is a serious issue worldwide, most of the reports come from Western countries. Dyspepsia is usually of structural type in developing countries, while in 
developed countries, FD is more prevalent., ${ }^{9,10}$

Although FD is not a life-threatening illness which usually does not require surgery and has not been shown to decrease survival, ${ }^{11}$ it disrupts the quality of life significantly and is associated with high rates of absence from work, poor efficiency, fewer activities around the house, and higher medical expenses, ${ }^{12,13}$ demonstrating that dyspepsia in general causes many mental health complications for the population. ${ }^{14}$

Many researchers have investigated the prevalence of dyspepsia in the Iranian population, but the results are not consistent. ${ }^{15-28}$ Thus, a coherent survey of all documents and combining them can provide a more comprehensive picture of the dimensions of this illness in Iranian community. The results of this meta-analysis can provide a more accurate estimate regarding the effect of therapies or risk factors for the illness or other results through combining different studies. ${ }^{29-31}$ The present research was carried out to assess the prevalence of dyspepsia in Iran.

\section{Materials and Methods}

\section{Study Protocol}

This research was carried out according to Metaanalyses Of Observational Studies in Epidemiology (MOOSE) guideline ${ }^{31}$ and reporting was performed according to the Preferred Reporting Items for Systematic Reviews and Meta-analysis (PRISMA) protocol. ${ }^{32}$ This meta-analysis was recorded in PROSPERO with code (CRD42019148610).

Two authors performed each step of the study independently. A third author resolved the inconsistencies in each step of the research and they were confirmed through discussion.

\section{Search Strategy and Selection of Studies}

A systematic search was conducted in July 2019 using international databases, including PubMed/Medline, Science Direct, Web of Science (ISI), Cochrane Library, Scopus, EMBASE, EBSCO, CINAHL, and national databases such as Magiran, Iranian Research Institute for Information Science and Technology (IranDoc), Scientific Information Database (SID), Barakat Knowledge Network System, Regional Information Center for Science and Technology (RICST), and the Iranian National Library, as well as Google Scholar.

The key terms are introduced in Table 1. We selected the "All Fields" option as the field of search. We combined the terms "descriptive", "population sentences" and "outcome" using the Boolean operator "AND".
To identify all potential studies, the reference lists of all found articles were manually reviewed.

\section{Inclusion and Exclusion Criteria}

The inclusion criteria were all cross-sectional articles on the prevalence of dyspepsia in Iran in both Persian and English. The exclusion criteria were: (1) non-adult samples (more than 10\% below the age of 15); (2) non-random samples; (3) non-Iranian subjects; (4) non-related subject; (5) sample size below 100 participants; (6) participants not from the general population or community; (7) case reports, case series, congresses, letters to the editor without quantitative data, reviews, and dissertations.

\section{Study Selection}

A reviewer (M.A) reviewed the titles and abstract of all detected reports and the full text was then assessed. The same action was repeated by a second researcher (M.K) independently. Eventually, any disagreement was discussed and resolved in the presence of a third researcher (M.S).

\section{Data Extraction}

The following data were recorded on a spreadsheet from each study: first author's name, publication date, year of study, mean age and standard deviation, how the data were collected, the diagnostic technique for dyspepsia, duration of symptoms for dyspepsia and prevalence of dyspepsia. At the end, the obtained data were imported into Excel.

For overlapping articles, we emailed the corresponding authors to determine the original article, and in case we did not receive any answer, we chose the article with the highest number of subjects for cases that overlapped.

\section{Quality Assessment}

Since all articles included the cross-sectional studies, the quality of the each study was assessed using the modified form of Newcastle Ottawa Scale. ${ }^{33}$ The study quality was categorized as follows: poor quality $=0-5$; medium quality $=6-8$; high quality $=9-10$. Articles with poor quality were excluded.

\section{Statistical Synthesis}

All analyses were performed using the Comprehensive Meta-Analysis software version 2. Heterogeneity was evaluated using $\mathrm{I}^{2}$ and chi-square tests. Heterogeneity was classified as follows: $\mathrm{I}^{2}$ values $0 \%-25 \%, 26 \%-50 \%, 50 \%-$ $75 \%$ and greater than $75 \%$ showed low, medium, high and very high heterogeneity, respectively, with $P$ value $<0.10$ considered statistically significant. ${ }^{34,35}$ In cases of

Table 1. Search terms

\begin{tabular}{ll}
\hline Search Term & Boolean Keywords \\
\hline Descriptive term & $\begin{array}{l}\text { frequency OR Incidence OR detection OR prevalence OR occurrence OR identification OR characterization OR } \\
\text { isolation OR investigation OR survey OR rate }\end{array}$ \\
\hline Population term & Iran \\
Outcome term & Dyspepsia OR Epigastric pain syndrome OR postprandial distress syndrome OR gastrointestinal diseases \\
\hline
\end{tabular}


high levels of heterogeneity among studies, we used the random effects model. The male/female odds ratio (OR) was used to show the impact of gender on dyspepsia. For this goal, the prevalence of dyspepsia in males, females and the total sample size was evaluated. Subgroup analysis was performed according to areas, population groups, study period, and diagnostic technique. Mixed effects metaregression was utilized to assess the relationship between study time and the prevalence of dyspepsia. Subgroup differences were also tested. ${ }^{36}$ Sensitivity analysis also was done. Publication bias was evaluated by an in-depth analysis of the funnel plot, using the proposed correlation tests of Egger and Begg's. ${ }^{37,38} P$ value less than 0.05 was considered statistically significant.

\section{Results}

\section{Search Results and Features}

Overall, 1460 articles were retrieved. Of these, 1446 studies were excluded because of: duplicate publications $(n=730)$, unrelated literature $(n=651)$, non-epidemiological studies $(\mathrm{n}=17)$, non-random samples or studies on children $(n=28)$, participants not from general population or community $(\mathrm{n}=18)$, as well as case reports, case series, congresses, letters to the editor without quantitative data, reviews, and dissertations $(n=2)$. Finally, fourteen studies remained for the meta-analysis (Figure 1). The mean age of samples was 37.41 years (95\% CI: 33.70-41.11). Three, two, one, two, four, one, and one studies were conducted in Tehran, Fars, East Azarbaijan, Hamadan, Isfahan, Kurdistan, and Kerman provinces, respectively. Ten, two, one, and one studies were conducted on the general population, healthcare workers, outpatient clinics, and blood donors, respectively. Four and ten studies had low and medium quality, respectively. Table 2 demonstrates the characteristics and general information of each study.

\section{Prevalence of Dyspepsia}

The prevalence of dyspepsia by combining fourteen studies with a sample size of 54118 subjects was 14.6\% (95\% CI: 9.6-21.7) (Figure 2A). The minimum and maximum

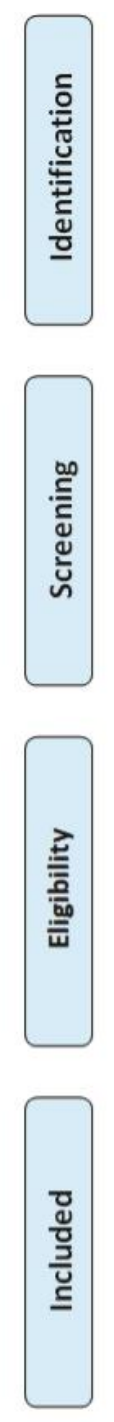

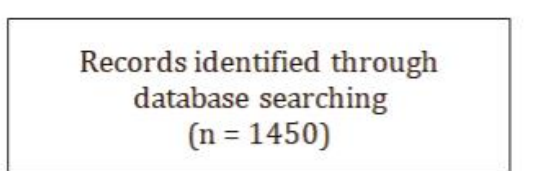
$(n=1450)$

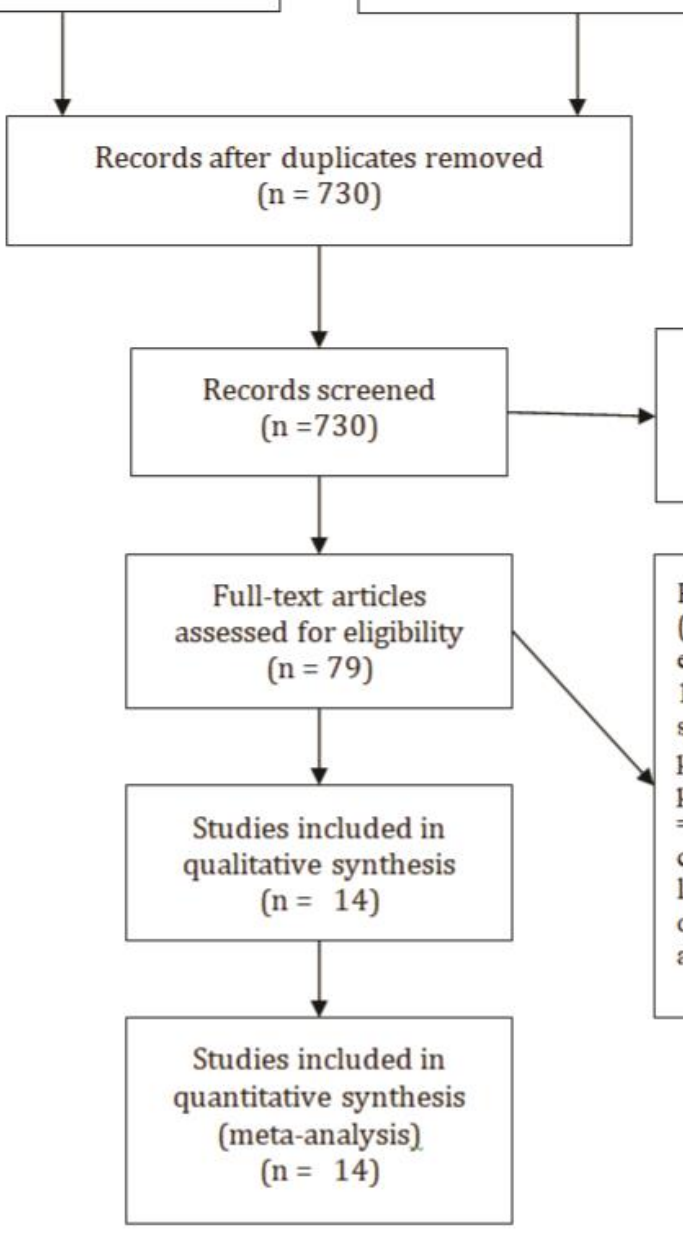

Additional records identified through other sources $(\mathrm{n}=10)$ related studies $(\mathrm{n}=651)$

Full-text articles excluded $(\mathrm{n}=65)$, with reasons: nonepidemiological studies $(\mathrm{N}=$ 17), non-random samples or studies on children $(\mathrm{N}=28)$, participants not from general population or community $(\mathrm{N}$ $=18$ ), as well as case reports, case series, congresses, letters to the editor without quantitative data, reviews, and dissertations $(\mathrm{N}=2)$.

Figure 1. Flowchart of Study Selection. 


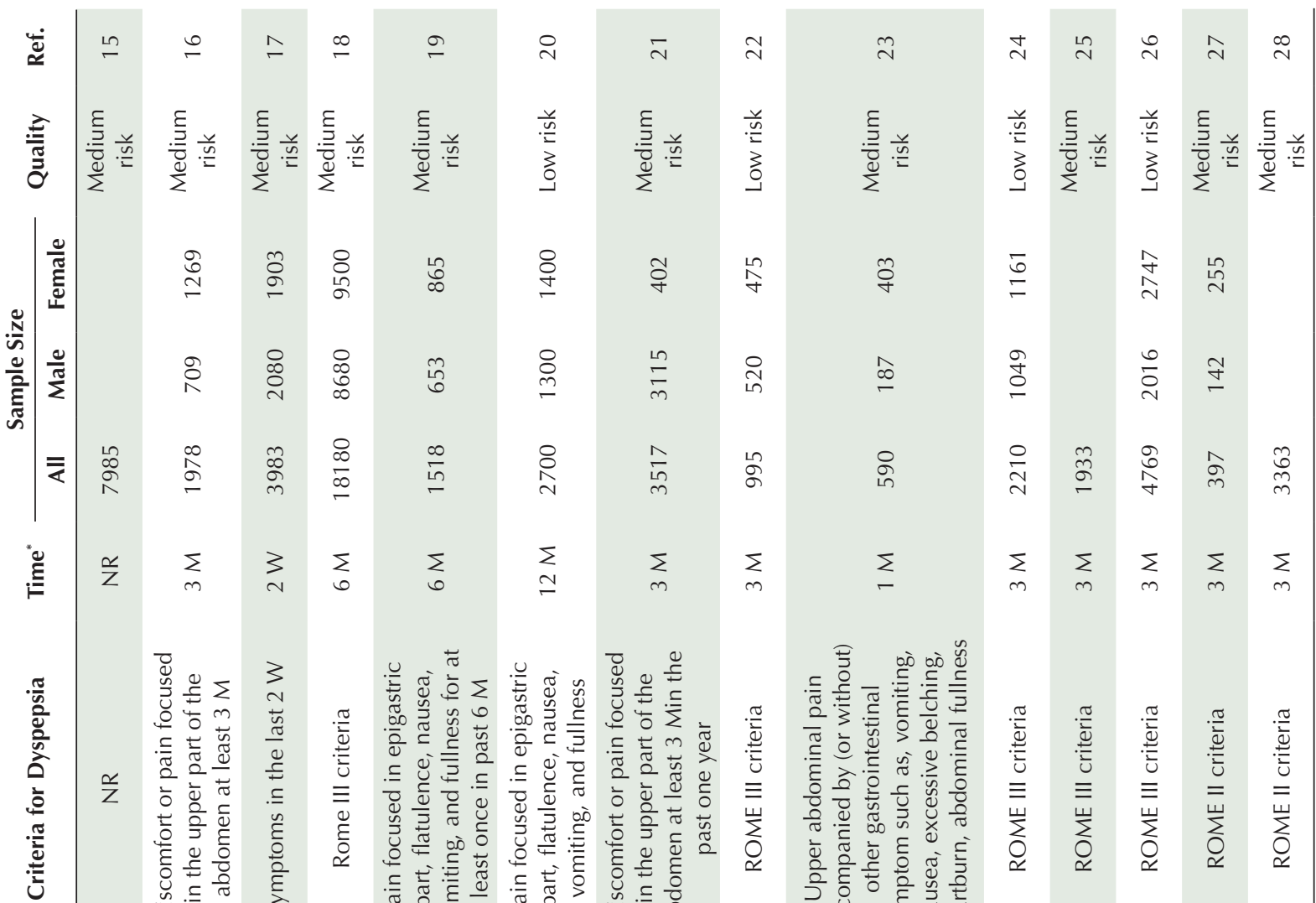

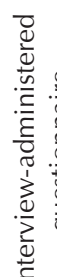

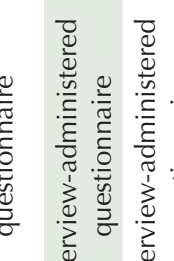

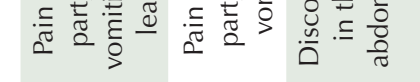
范 है

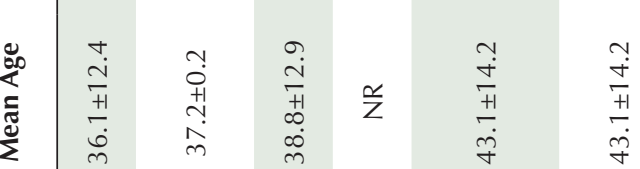
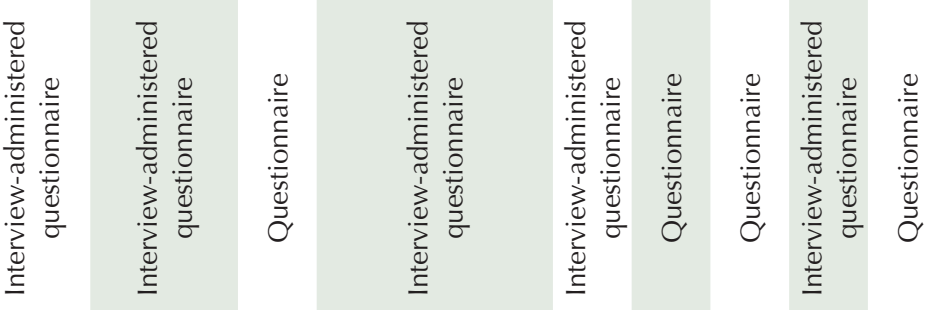

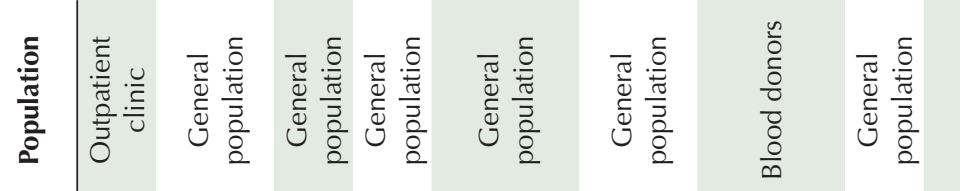

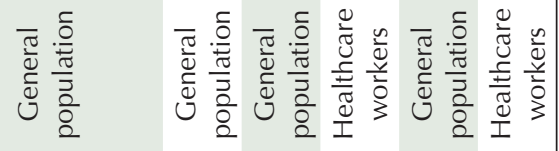

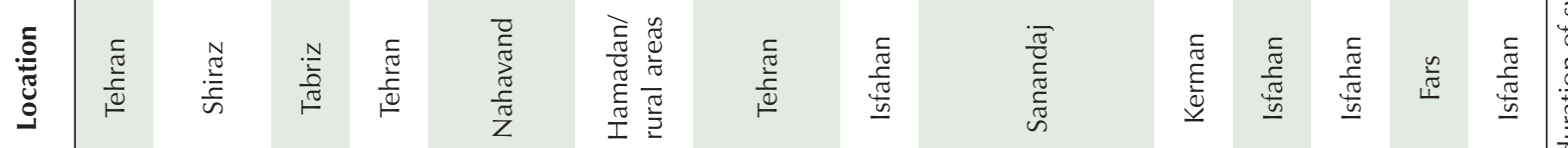


prevalence of dyspepsia pertained to the studies by Moghimi-Dehkordi et $\mathrm{al}^{18}(2.2 \%)$ and Yazdanpanah et $\mathrm{al}^{23}$ (54.6\%), respectively. The prevalence of dyspepsia, after removing two high-prevalence studies (Yazdanpanah et $\mathrm{al}^{23}$ and Masoumi et $\mathrm{al}^{27}$ ), was $11.3 \%$ (95\% CI: 7.4-16.7) (Figure 2B).

\section{Prevalence of all Types of Dyspepsia}

The prevalence rates of ulcer-like dyspepsia in five studies with a sample size of 8,000 subjects, dysmotility-like dyspepsia in five studies with a sample size of 8000 , and unspecified dyspepsia in four studies with a sample size of 4,483 were estimated to be $12.1 \%$ (95\% CI: $5.2-25.7$ ), 9.7\% (95\% CI: 4.9-18.4) and 17.0\% (95\% CI: 7.8-33.4), respectively (Figure 3).

\section{Gender-Specific Prevalence of Dyspepsia}

The prevalence of dyspepsia in Iranian males (in eleven studies with a sample size of 20,541) and females (in eleven studies with a sample size of 20380) was estimated at $11.1 \%$ (95\% CI: $6.3-18.8$ ) and $17.8 \%$ (95\% CI: $10.0-$ 29.7), respectively. The females/males OR for dyspepsia was estimated at 1.52 (95\% CI: $1.32-1.75, P<0.001$ ), which was significant (Figure 4C).

\section{Subgroup Analysis for Dyspepsia}

The prevalence of dyspepsia according to geographic area in Center, South, North and West of Iran was found to be 9.1\% (95\% CI: $5.5-14.9), 29.6 \%$ (95\% CI: 16.4-47.5), 2.9\% (95\% CI: $2.4-3.5)$ and 30.2\% (95\% CI: 15.1-51.3), respectively (Figure 5A). The prevalence of dyspepsia based on population groups in blood donors, general population, healthcare workers and outpatient clinics was estimated to be $8.9 \%$ (95\% CI: 8.0-9.9), $15.7 \%$ (95\% CI: 7.9-28.7), 15.1\% (95\% CI: 14.1-16.1), and 11.4\% (95\% CI: 10.7-12.1), respectively (Figure 5B). The prevalence of dyspepsia based on duration of symptoms in $\leq 1,3,6$ and 12 months was estimated to be $15.9 \%$ (95\% CI: $0.5-87.6$ ),

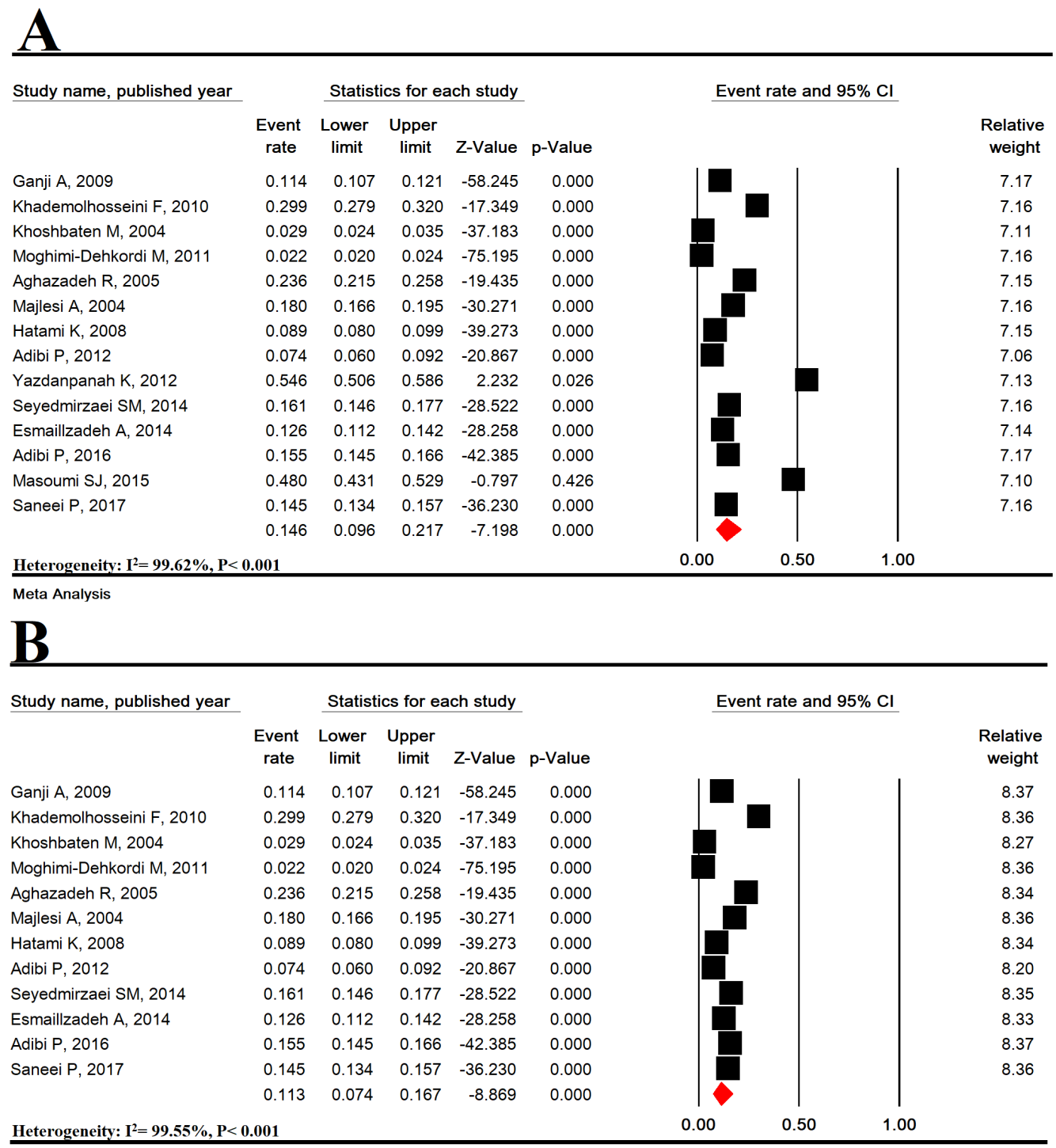

Figure 2. Prevalence of Dyspepsia in Iran. 


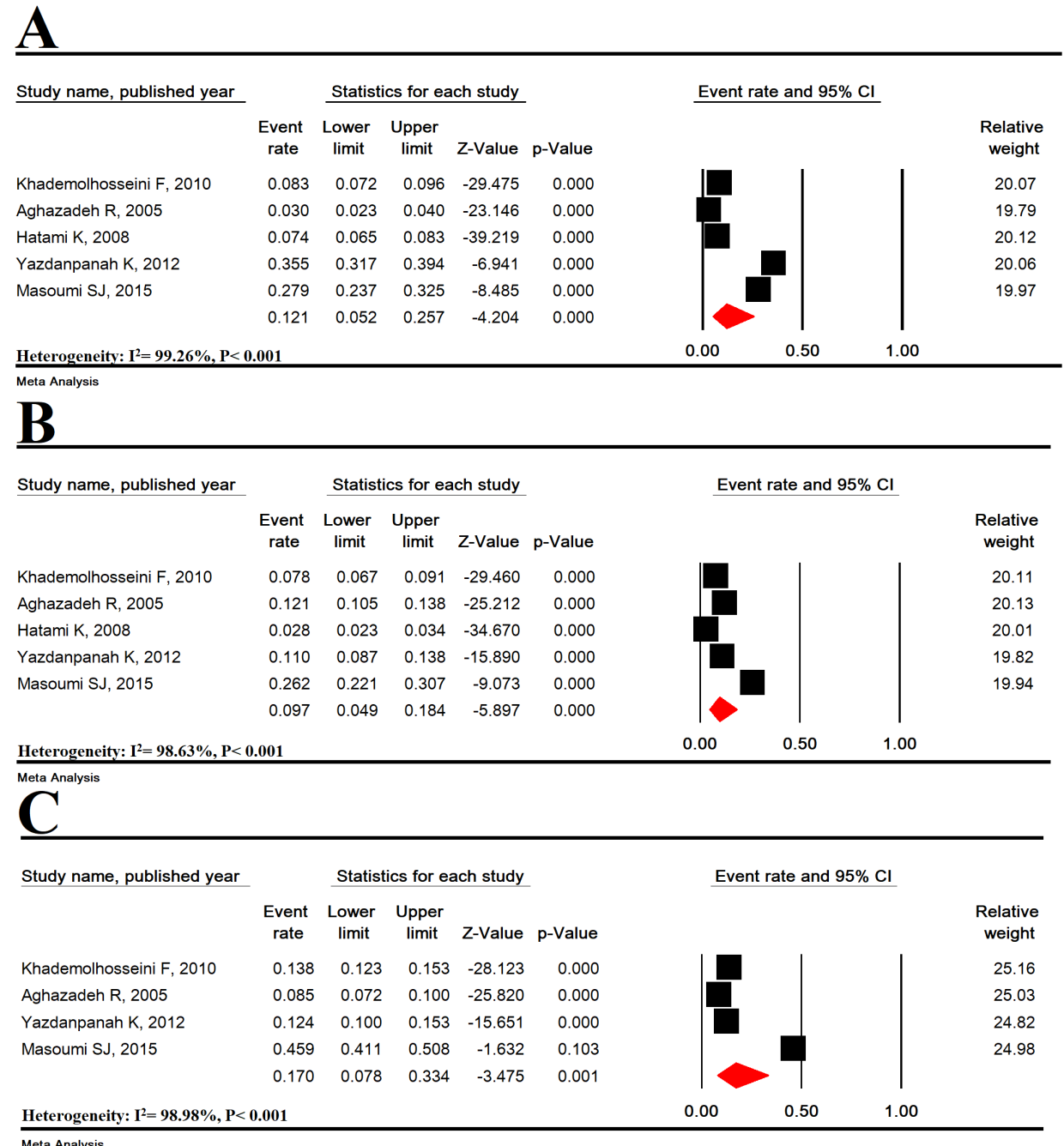

Heterogeneity: $I^{2}=98.98 \%, P<0.001$

Figure 3. Prevalence of Ulcer-like (A), Dysmotility-Like (B) and Unspecified (C) Dyspepsia.

16.7\% (95\% CI: $11.8-23.3), 7.7 \%$ (95\% CI: 0.6-51.9), and $18.0 \%$ (95\% CI: 16.6-19.5), respectively (Figure 5C). The prevalence of dyspepsia based on the diagnostic technique in dyspepsia symptoms, ROME II and ROME III was estimated to be $17.8 \%$ (95\% CI: $9.0-32.1$ ), $28.3 \%$ (95\% CI: 7.0-67.5), and 9.0\% (95\% CI: 3.8-19.6), respectively (Figure 5D). The prevalence of dyspepsia based on data collection using interviews, interview-administered questionnaires and questionnaires was estimated to be 11.4\% (95\% CI: 10.7-12.1), 16.3\% (95\% CI: 7.9-30.8), and 12.4\% (95\% CI: 10.1-15.2), respectively (Figure 5E).

Differences in subgroup analysis based on geographic area $(P<0.001)$, population groups $(P<0.001)$ and duration of symptoms $(P<0.001)$ were significant, but differences in subgroup analysis based on the method of data collection $(P=0.458)$ and diagnostic technique $(P=0.276)$ were not significant (Figure 5).

\section{Meta-regression Model}

No significant relationship was observed between year of study, the total prevalence of dyspepsia (Meta-regression: $0.086,95 \% \mathrm{CI}:-0.037$ to $0.209, P=0.172)$, and the prevalence of dyspepsia in men (Meta-regression: 0.136,
95\% CI: -0.036 to $0.308, P=0.121$ ) and women (Metaregression: $0.100,95 \% \mathrm{CI}$ : -0.096 to $0.088, P=0.296$ ) (Figure S1, Supplementary file 1).

\section{Sensitivity Analysis}

Sensitivity analysis showed that the total estimates are robust for prevalence of dyspepsia and for males and females (Figure S2, Supplementary file 1).

\section{Publication Bias}

Publication bias was not significant according to Begg's $(P=0.912)$ and Egger's $(P=0.669)$ test (Figure S3, Supplementary file 1).

\section{Discussion}

This research is the first meta-analysis addressing the prevalence of dyspepsia in Iranian people. The prevalence of dyspepsia was estimated to be $14.6 \%$ based on 14 studies. In a review study, the global prevalence of dyspepsia was found to be $31 \%$, while indicating different prevalence in various areas such as North America (30\%), South America (29\%), North Europe (29\%), South Europe (37\%), South Asia (49\%), Southeast Asia (27\%), and Australia 


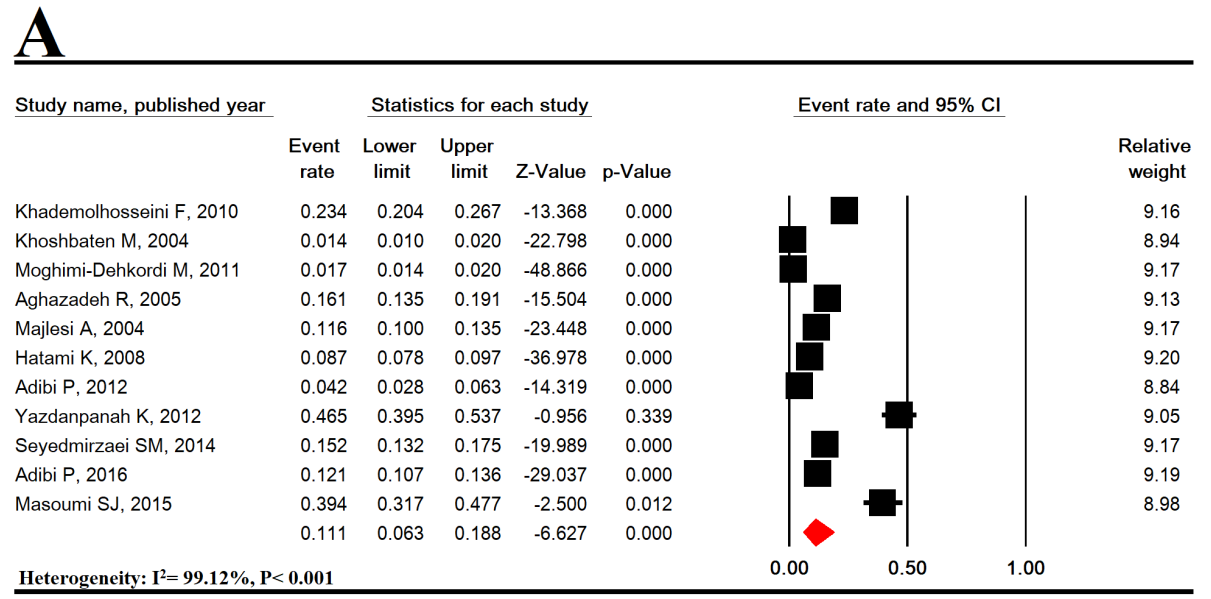

Heterogeneity: $\mathrm{I}^{2}=\mathbf{9 9 . 1 2 \% , P < 0 . 0 0 1}$

\section{Meta Analysis}

B

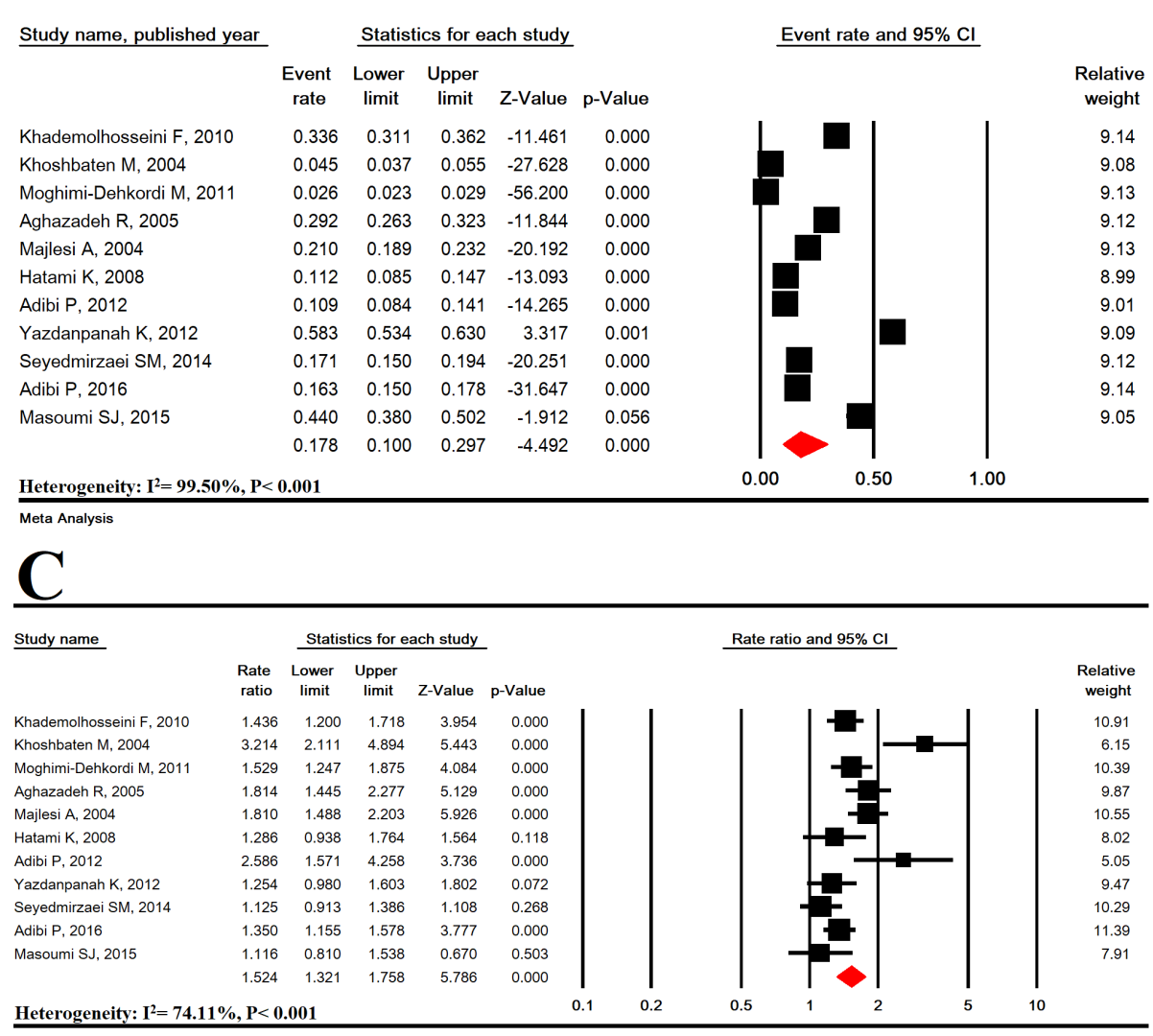

Figure 4. Prevalence of Dyspepsia in Iranian Males (A) and Females (B) and the Male to Female Odds Ratio for Dyspepsia (C).

$(25 \%) .{ }^{39}$ Various meta-analyses have been conducted on the prevalence of dyspepsia in special disorders, and the prevalence of dyspepsia was $43.9 \%$ in GERD patients, ${ }^{40}$ $65.7 \%$ in patients with cholecystolithiasis ${ }^{41}$ and $9.55 \%$ after acute gastroenteritis. ${ }^{42}$ In another review, the global unexamined dyspepsia prevalence was $29 \%$. However, in studies in the Middle East (including Iran), it was reported to be $15 \% .{ }^{43}$ A systematic review and meta-analysis study in Iranian population the daily, weekly, monthly, and overall prevalence of GERD symptoms was 5.64\%, 12.50\%, $18.62 \%$ and $43.07 \%$, respectively. ${ }^{44}$

The present meta-analysis has many strengths. We utilized a universal and repeated search to increase the ability to detect all related papers. All phases of the study were independently carried out by two authors, and the disputes were resolved by a third author. We made contact with the first or corresponding authors to make sure we omit duplicate publications, and to gain additional data in certain cases. A random effects model was used to merge the data to prepare dependable estimates of dyspepsia prevalence. We also performed subgroup analysis to identify the reason for heterogeneity and to assess publication bias. Finally, the limitations of the present study are associated with the search in local databases, 
A

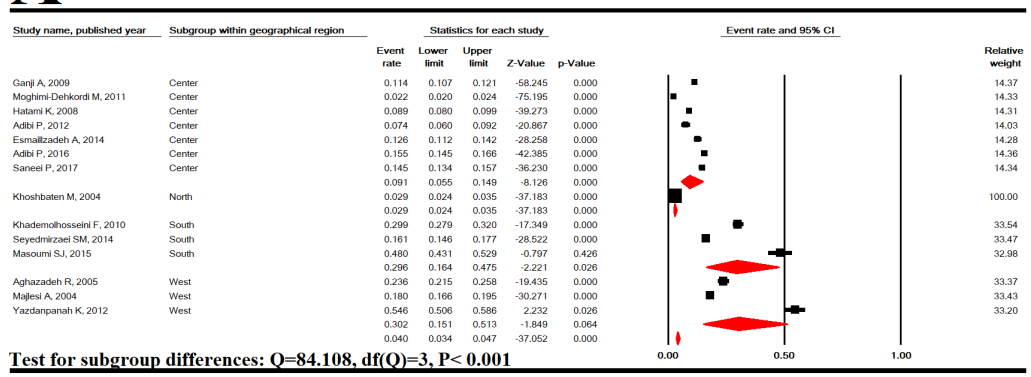

B

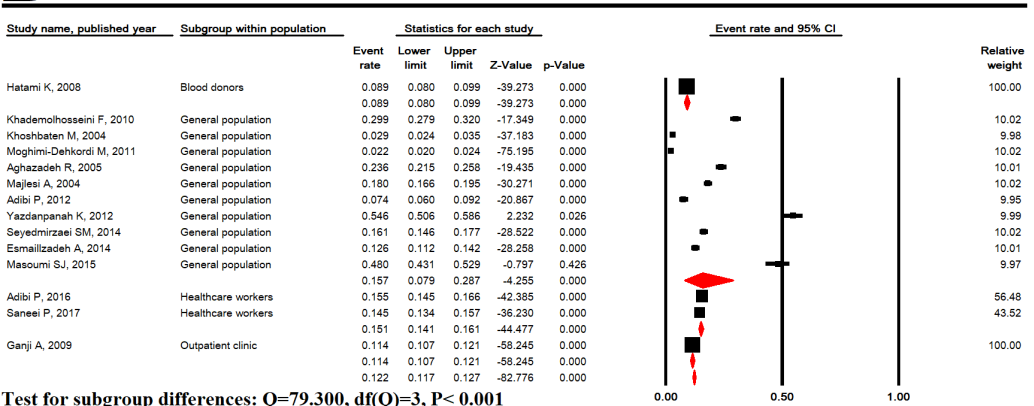

Test for subgroup differences: $Q=79.300, \mathrm{df}(\mathrm{Q})=3, \mathrm{P}<\mathbf{0 . 0 0 1}$

C

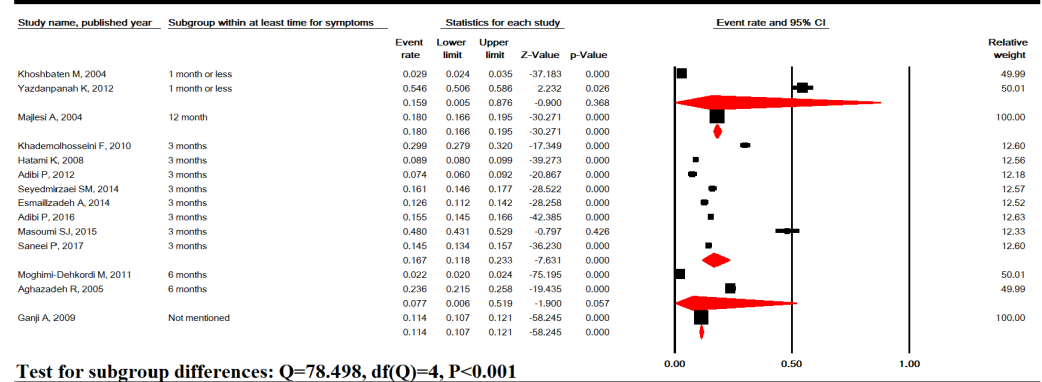

Test for subgroup differences: $Q=78.498, \mathrm{df}(Q)=4, P<0.001$

D

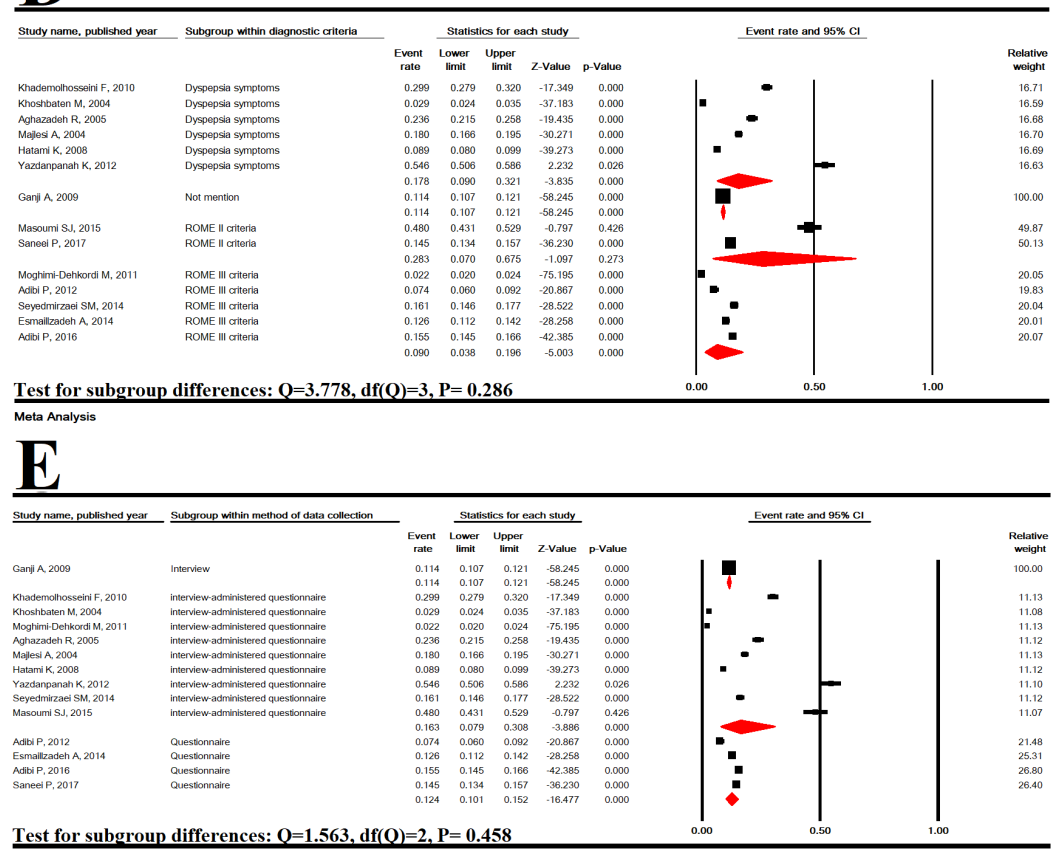

Figure 5. Subgroup Analysis Differences Based on Geographic Area (A), Population Groups (B) and at Least for Duration of Symptoms (C), Method of Data Collection (D) and Diagnostic Technique (E). 
which lack the potential for combined search. We also excluded studies that were performed on special patients or had non-random sample size, so that the estimate would be attributable to the general population. Moreover, the limitations of the initial studies were about data collection, as some of them used questionnaires and some used interviews or both. Of course, more personal methods, such as interviews, which are the dominant methods in studies included in the meta-analysis, may overestimate the prevalence, but more impersonal techniques, like completing a questionnaire form on a website, might be more accurate. The absence of articles that show the prevalence of dyspepsia for some geographical regions such as eastern and northern Iran may be considered as other limitations. Moreover, there was significant heterogeneity across all studies in our analysis, and considering the accessible data, we could attribute this difference to the geographic area $(P<0.001)$, population groups $(P<0.001)$, and duration of symptoms $(P<0.001)$ by subgroup analysis. Nevertheless, it seems that due to other cultural differences between the population groups, such as diversities in lifestyle and ethnicity (given that Iranian people have comprise several ethnicities with different cultures), ${ }^{43}$ the accuracy and robustness of data collection could not be evaluated based on the available data.

The combination of epidemiological studies in Iran in this research showed that the prevalence of dyspepsia was significantly higher in women compared to men $(\mathrm{OR}=1.52)$. In a meta-analysis at the global level, the unexamined dyspepsia prevalence was significantly higher in women compared to men in the Middle East $(\mathrm{OR}=1.91)$. However, it was not significant in African countries, Southeast Asia, South America, Central America and Australia. ${ }^{45}$

In the present study, the lowest incidence of dyspepsia based on the diagnostic technique was observed for the ROME III criteria (9\%). These criteria were published 12 years ago for defining dyspepsia ${ }^{46}$; few studies have evaluated these criteria and no significant difference was observed between ROME III and previous criteria for dyspepsia in one study. ${ }^{47}$

The prevalence of dyspepsia according to the study year showed an increasing trend in the meta-regression model, although it was not significant. This increase might be due to the fact that dyspepsia has no impact on mortality. ${ }^{11,48}$

Evaluating publication bias in the results of this study showed that bias had no impact on these studies. The bias is often created in these articles, since such articles are more probable to be printed because of their positive results. However, no such bias was found about dyspepsia in this study. ${ }^{49}$

In conclusion, the prevalence of dyspepsia in Iran is relatively high (14.6\%) but is lower than worldwide estimates.

\section{Authors' Contribution}

MA, MK, AA, and ZJ MS performed data extraction. MA performed statistical analysis. MA and RR wrote the manuscript. MA, MK, AA, and ZJ, RR and MS performed the literature search and quality evaluation. MA, MK, AA, and ZJ, RR and MS revised the manuscript. All authors read and approved the final manuscript.

\section{Conflict of Interest Disclosures}

The authors declare no conflict of interests.

Funding

Ilam University of Medical Sciences.

Ethical Statement

Not applicable.

\section{Acknowledgment}

The authors would like to thank the Clinical Research Development Unit of Baqiyatallah Hospital, Tehran, Iran, for guidance and advice.

\section{Supplementary Materials}

Supplementary file 1 contains Figures S1-S3.

\section{References}

1. Zagari RM, Law GR, Fuccio L, Cennamo V, Gilthorpe MS, Forman D, et al. Epidemiology of and subgroups in the Italian general population: an endoscopic study. Gastroenterology. 2010;138(4):1302-11. doi: 10.1053/j. gastro.2009.12.057.

2. Geeraerts B, Tack J. Functional dyspepsia: past, present, and future. J Gastroenterol. 2008;43(4):251-5. doi: 10.1007/ s00535-008-2167-8.

3. Tosetti C, Stanghellini V, Corinaldesi R. The Rome II Criteria for patients with functional gastroduodenal disorders. J Clin Gastroenterol. 2003;37(1):92-3. doi: 10.1097/00004836-200307000-00028.

4. Stanghellini V, Chan FK, Hasler WL, Malagelada JR, Suzuki $\mathrm{H}$, Tack J, et al. Gastroduodenal disorders. Gastroenterology. 2016;150(6):1380-92. doi: 10.1053/j.gastro.2016.02.011.

5. Greydanus MP, Vassallo M, Camilleri M, Nelson DK, Hanson RB, Thomforde GM. Neurohormonal factors in functional dyspepsia: insights on pathophysiological mechanisms. Gastroenterology. 1991;100(5 Pt 1):1311-8.

6. Hosseinzadeh M, Khosravi A, Saki K, Ranjbar R. Evaluation of Helicobacter pylori infection in patients with common migraine headache. Arch Med Sci. 2011;7(5):844-9. doi: 10.5114/aoms.2011.25560.

7. Ranjbar R, Khamesipour F, Jonaidi-Jafari N, Rahimi E. Helicobacter pylori isolated from Iranian drinking water: $\operatorname{vacA}, \operatorname{cag} \mathrm{A}, \mathrm{ice} \mathrm{A}$, oipA and babA2 genotype status and antimicrobial resistance properties. FEBS Open Bio. 2016 ;6(5):433-41.

8. Ranjbar R, Behzadi P, Farshad S. Advances in diagnosis and treatment of Helicobacter pylori infection. Acta Microbiol Immunol Hung. 2017;64(3):273-92. doi: 10.1556/030.64.2017.008 .

9. Kim SE, Park HK, Kim N, Joo YE, Baik GH, Shin JE, et al. Prevalence and risk factors of functional dyspepsia: 
a nationwide multicenter prospective study in Korea. J Clin Gastroenterol. 2014;48(2):e12-8. doi: 10.1097/ MCG.0b013e31828f4bc9.

10. Mahadeva S, Goh KL. Epidemiology of functional dyspepsia: a global perspective. World J Gastroenterol. 2006;12(17):2661-6. doi: 10.3748/wjg.v12.i17.2661.

11. Ghoshal UC, Singh R, Chang FY, Hou X, Wong BC, Kachintorn $U$. Epidemiology of uninvestigated and functional dyspepsia in Asia: facts and fiction. J Neurogastroenterol Motil. 2011;17(3):235-44. doi: 10.5056/ jnm.2011.17.3.235.

12. Camilleri M, Dubois D, Coulie B, Jones M, Kahrilas PJ, Rentz AM, et al. Prevalence and socioeconomic impact of upper gastrointestinal disorders in the United States: results of the US Upper Gastrointestinal Study. Clin Gastroenterol Hepatol. 2005;3(6):543-52. doi: 10.1016/ s1542-3565(05)00153-9.

13. Brook RA, Kleinman NL, Choung RS, Melkonian AK, Smeeding JE, Talley NJ. Functional dyspepsia impacts absenteeism and direct and indirect costs. Clin Gastroenterol Hepatol. 2010;8(6):498-503. doi: 10.1016/j. cgh.2010.03.003.

14. Lacy BE, Weiser KT, Kennedy AT, Crowell MD, Talley NJ. Functional dyspepsia: the economic impact to patients. Aliment Pharmacol Ther. 2013;38(2):170-7. doi: 10.1111/ apt.12355.

15. Ganji A, Malekzadeh F, Safavi M, Nassri-Moghaddam S, Nourie M, Merat S, et al. Digestive and liver disease statistics in Iran. Middle East J Dig Dis. 2009;1(2):56-62.

16. Khademolhosseini F, Mehrabani D, Zare N, Salehi M, Heydari S, Beheshti M, et al. Prevalence of dyspepsia and its correlation with demographic factors and lifestyle in Shiraz, southern Iran. Middle East J Dig Dis. 2010;2(1):24-30.

17. Khoshbaten M, Hekmatdoost A, Ghasemi H, Entezariasl M. Prevalence of gastrointestinal symptoms and signs in northwestern Tabriz, Iran. Indian J Gastroenterol. 2004;23(5):168-70.

18. Moghimi-Dehkordi B, Vahedi M, Khoshkrood Mansoori B, Kasaeian A, Safaee A, Habibi M, et al. Economic burden of gastro-oesophageal reflux disease and dyspepsia: a community-based study. Arab J Gastroenterol. 2011;12(2):86-9. doi: 10.1016/j.ajg.2011.03.005.

19. Aghazadeh R, Mohammad Alizadeh AH, Ansari S, Ranjbar M, Hosseinnejad Yazdi M, Honarkar Z, et al. Epidemiology of dyspepsia in Nahavand, Iran. Res Med. 2005;29(1):33-6. [Persian].

20. Majlesi A, Mani Kashani K, Karimi MM. Prevalence of dyspepsia in rural districts of Hamadan city in 2002. Avicenna J Clin Med. 2004;11(1):47-50. [Persian].

21. Hatami K, Pourshams A, Azimi K, Sarrafi M, Mehrabani $\mathrm{M}$, Mostajabi P, et al. Dyspepsia, gastroesophageal reflux disease and irritable bowel syndrome among blood donors. Govaresh. 2003;8(4):138-46. [Persian].

22. Adibi P, Behzad E, Shafieeyan M, Toghiani A. Upper functional gastrointestinal disorders in young adults. Med Arh. 2012;66(2):89-91. doi: 10.5455/medarh.2012.66.89-91.

23. Yazdanpanah K, Moghimi N, Yousefinejad V, Ghaderi E, Azizi A, Nazem SF. Dyspepsia prevalence in general population aged over 20 in the west part of Iran. J Pak Med Assoc. 2012;62(7):672-6.

24. Seyedmirzaei SM, Haghdoost AA, Afshari M, Dehghani A. Prevalence of dyspepsia and its associated factors among the adult population in southeast of Iran in 2010. Iran Red Crescent Med J. 2014;16(11):e14757. doi: 10.5812/ ircmj.14757.

25. Esmaillzadeh A, Hassanzadeh Keshteli A, Tabesh M, Feizi A, Adibi P. Smoking status and prevalence of upper gastrointestinal disorders. Digestion. 2014;89(4):282-90. doi: $10.1159 / 000358169$.

26. Adibi P, Hassanzadeh Keshteli A, Saneei M, Saneei P, Savabi O, Esmaillzadeh A. Relationship between tooth loss, functional dyspepsia and gastro-esophageal reflux disorder among Isfahani adults. Arch Iran Med. 2016;19(2):123-30.

27. Masoumi SJ, Mehrabani D, Moradi F, Zare N, Saberi-Firouzi M, Mazloom Z. The prevalence of dyspepsia symptoms and its correlation with the quality of life among Qashqai Turkish migrating nomads in Fars province, southern Iran. Pak J Med Sci. 2015;31(2):325-30. doi: 10.12669/ pjms.312.6956.

28. Saneei P, Hajishafiee M, Esmaillzadeh A, Hassanzadeh Keshteli A, Roohafza H, Afshar H, et al. Association between healthy lifestyle score and upper gastrointestinal disorders in Iranian adults. J Gorgan Univ Med Sci. 2017;19(1):54-66. [Persian].

29. Azami M, Jaafari Z, Masoumi M, Shohani M, Badfar G, Mahmudi L, et al. The etiology and prevalence of urinary tract infection and asymptomatic bacteriuria in pregnant women in Iran: a systematic review and meta-analysis. BMC Urol. 2019;19(1):43. doi: 10.1186/s12894-019-0454-8.

30. Sayehmiri K, Tavan H, Sayehmiri F, Mohammadi I, V Carson K. Prevalence of epilepsy in iran: a meta-analysis and systematic review. Iran J Child Neurol. 2014;8(4):9-17. doi:10.22037/ijcn.v8i4.5880.

31. Stroup DF, Berlin JA, Morton SC, Olkin I, Williamson GD, Rennie D, et al. Meta-analysis of observational studies in epidemiology: a proposal for reporting. JAMA. 2000;283(15):2008-12. doi: 10.1001/jama.283.15.2008.

32. Shamseer L, Moher D, Clarke M, Ghersi D, Liberati A, Petticrew M, et al. Preferred reporting items for systematic review and meta-analysis protocols (PRISMA-P) 2015: elaboration and explanation. BMJ. 2015;350:g7647. doi: 10.1136/bmj.g7647.

33. Wells GA, Shea B, O'Connell D, Peterson J, Welch V, Losos $\mathrm{M}$, et al. The Newcastle-Ottawa Scale (NOS) for Assessing the Quality of Nonrandomized Studies in Meta-Analyses. 2011. Available from: http://www.ohri.ca/programs/ clinical_epidemiology/oxford.asp. Cited 2012 Nov 25.

34. Higgins JPT, Green S. Cochrane Handbook for Systematic Reviews of Interventions Version 5.1.0. Available from: http://handbook.cochrane.org/.

35. Ades AE, Lu G, Higgins JP. The interpretation of randomeffects meta-analysis in decision models. Med Decis Making. 2005;25(6):646-54. doi: 10.1177/0272989x05282643.

36. Higgins JP, Thompson SG, Deeks JJ, Altman DG. Measuring inconsistency in meta-analyses. BMJ. 2003;327(7414):55760. doi: 10.1136/bmj.327.7414.557.

37. Begg CB, Mazumdar M. Operating characteristics of a rank correlation test for publication bias. Biometrics. 1994;50(4):1088-101.

38. Egger M, Davey Smith G, Schneider M, Minder C. Bias in meta-analysis detected by a simple, graphical test. Bmj. 1997;315(7109):629-34. doi: 10.1136/bmj.315.7109.629.

39. Nakada K, Hongo M, Harasawa S, Mine T, Sasaki I, Matsueda K, et al. The worldwide prevalence of dyspepsia: 
systematic review and meta-analysis. Syst Rev. 2014;3:71. doi: 10.1186/2046-4053-3-71.

40. Eusebi LH, Ratnakumaran R, Bazzoli F, Ford AC. Prevalence of dyspepsia in individuals with gastroesophageal refluxtype symptoms in the community: a systematic review and meta-analysis. Clin Gastroenterol Hepatol. 2018;16(1):3948.e1. doi: 10.1016/j.cgh.2017.07.041.

41. Latenstein CSS, de Jong JJ, Eppink JJ, Lantinga MA, van Laarhoven C, de Reuver PR, et al. Prevalence of dyspepsia in patients with cholecystolithiasis: a systematic review and meta-analysis. Eur J Gastroenterol Hepatol. 2019;31(8):92834. doi: 10.1097/meg.0000000000001463.

42. Futagami S, Itoh T, Sakamoto C. Systematic review with meta-analysis: post-infectious functional dyspepsia. Aliment Pharmacol Ther. 2015;41(2):177-88. doi: 10.1111/ apt.13006.

43. Azami M, Moslemirad M, YektaKooshali MH, Rahmati S, Soleymani A, Bigdeli Shamloo MB, et al. Workplace violence against Iranian nurses: a systematic review and meta-analysis. Violence Vict. 2018;33(6):1148-75. doi: 10.1891/0886-6708.33.6.1148.

44. Karimian M, Nourmohammadi H, Salamati M, Hafezi Ahmadi MR, Kazemi F, Azami M. Epidemiology of gastroesophageal reflux disease in Iran: a systematic review and meta-analysis. BMC Gastroenterol. 2020;20(1):297. doi: 10.1186/s12876-020-01417-6.

45. Ford AC, Marwaha A, Sood R, Moayyedi P. Global prevalence of, and risk factors for, uninvestigated dyspepsia: a meta-analysis. Gut. 2015;64(7):1049-57. doi: 10.1136/ gutjnl-2014-307843.

46. Tack J, Talley NJ, Camilleri M, Holtmann G, Hu P, Malagelada JR, et al. Functional gastroduodenal disorders. Gastroenterology. 2006;130(5):1466-79. doi: 10.1053/j. gastro.2005.11.059.

47. Ford AC, Bercik P, Morgan DG, Bolino C, Pintos-Sanchez MI, Moayyedi P. The Rome III criteria for the diagnosis of functional dyspepsia in secondary care are not superior to previous definitions. Gastroenterology. 2014;146(4):93240; quiz e14-5. doi: 10.1053/j.gastro.2014.01.014.

48. Ford AC, Mahadeva S, Carbone MF, Lacy BE, Talley NJ. Functional dyspepsia. Lancet. 2020;396(10263):1689-702. doi: 10.1016/s0140-6736(20)30469-4.

49. Sayehmiri K, Azami M, Nikpey S, Borji M, Sayehmiri F. Hepatitis B vaccination coverage in health personnel of Iran: a systematic review and meta-analysis study. Iran J Epidemiol. 2015;11(3):1-10. [Persian]. 